\title{
The effects of pentoxifylline on skeletal muscle contractility and neuromuscular transmission during hypoxia
}

\author{
Fat ma Simsek-Duran, Mert Ertunc ${ }^{1}$, Rustu Onur ${ }^{1}$
}

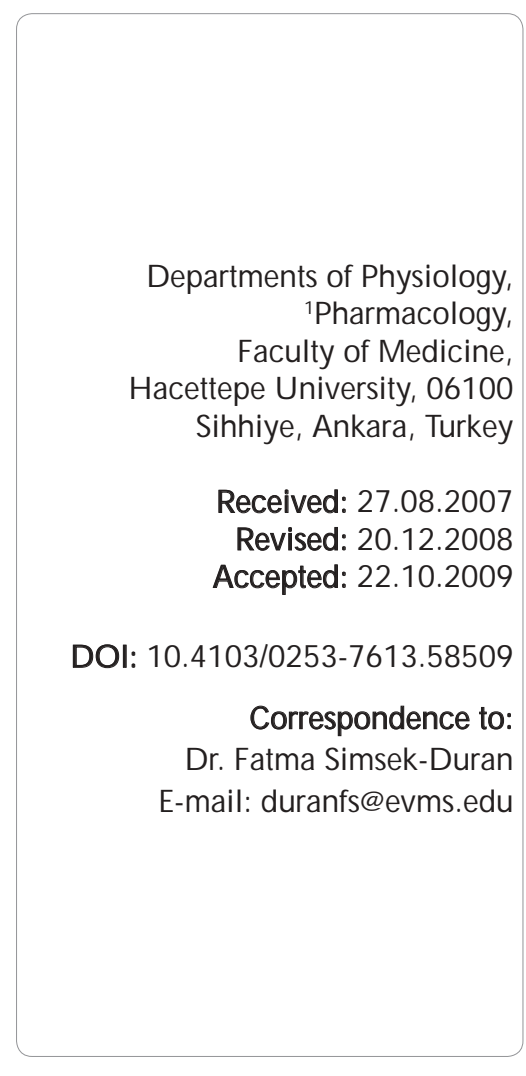

\begin{abstract}
O bjectives: The objective of this study was to investigate the effects of pentoxifylline (PTX), a drug that is mainly used for indications related to tissue hypoxia, on hypoxia-induced inhibition of skeletal muscle contractility and neuromuscular transmission in mice. We hypothesized that chronic PTX treatment alters skeletal muscle contractility and hypoxia-induced dysfunction.

Materials and M ethods: M ice were treated with $50 \mathrm{mg} / \mathrm{kg} \mathrm{PTX}$ or saline intraperitoneally for a week. Following ether anesthesia, diaphragm muscles were removed; isometric muscle contractions and action potentials were recorded. Time to reach neuromuscular blockade and the rate of recovery of muscle contractility were assessed during hypoxia and re-oxygenation.

Results: The PTX group displayed $90 \%$ greater twitch amplitudes $(P<0.01)$. Hypoxia depressed twitch contractions and caused neuromuscular blockade in both groups. However, neuromuscular blockade occurred earlier in PTX-treated animals $(P<0.05)$. Muscle contractures developed during hypoxia were more pronounced in the PTX group $(P<0.05)$. Re-oxygenation reduced contracture and indirect muscle contractions resumed. The rate of recovery of contractions was faster $(P<0.05)$ and the amplitude of contractions was greater $(P<0.01)$ in the PTX group. PTX treatment increased amplitude $(P<0.05)$ and shortened action potential $(P<0.05)$ without altering resting membrane potential, excitation threshold, and neurotransmitter release.

Conclusion: Chronic PTX treatment increases diaphragm contractility, but amplifies hypoxia-induced contractile dysfunction in mice. These results may implicate important clinical consequences for clinical usage of PTX in hypoxia-related conditions.
\end{abstract}

KEY WORDS: Contractility, hypoxia, neuromuscular transmission, pentoxifylline

\section{Introduction}

Hypoxia inhibits skeletal muscle contractility and causes neuromuscular blockade. Intracellular mechanisms of hypoxia-induced damage in skeletal muscle have not been fully elucidated. Impaired calcium $\left(\mathrm{Ca}^{2+}\right)$ buffering and decreased adenosine-5'-triphosphate (ATP) production in myocytes are known to participate in hypoxia-induced impairment of muscle function. ${ }^{[1]}$

Pentoxifylline (PTX), a xanthine-derived phosphodiesterase (PDE) inhibitor, is known to increase intracellular cyclic-adenosine monophosphate (cAMP) levels. ${ }^{[2]}$ An increase in cAMP levels in muscle fibers results in the activation of protein kinase A (PKA) and facilitates synaptic transmission in the mammalian neuromuscular junction (NMJ ). ${ }^{[3]}$ Recent data showed that PTX administration decreases plasma levels of pro-inflammatory cytokines, such as tumor necrosis factor alpha(TNF- $\alpha)$, interleukin (IL)-1 $\beta, \mathrm{IL}-10,{ }^{[4]}$ and down-regulates their production. ${ }^{[5]}$ Recently, cytokines have been shown to impair contractile response in the skeletal muscle probably by affecting the nitrergic pathway or nitric oxide (NO) production. ${ }^{[6,7]}$

PTX exerts beneficial effects on the treatment of peripheral circulatory insufficiency. A recent study has demonstrated that PTX attenuates ischemia reperfusion injury in the skeletal muscle and other tissues by decreasing neutrophil adhesion to endothelial cells, reactive oxygen species production, and platelet activation. ${ }^{[8]}$ However, the effects of PTX treatment on muscle contractility and on hypoxia-induced contractile dysfunction have not been studied. This study was designed to evaluate the effects of chronic PTX treatment on the contractile properties of the skeletal muscle and hypoxia-induced contractile impairment. We hypothesized that chronic PTX treatment alters skeletal muscle contractility and hypoxia-induced dysfunction. 


\section{Materials and Methods}

Mice, weighing between 30 and $35 \mathrm{~g}$, were obtained from the Hacettepe University, Department of Pharmacology Animal Breeding Unit. Animals were housed in communal cages with food and water available ad libitum, and exposed to a $12 \mathrm{~h}$ light/dark cycle with the room temperature maintained at $21^{\circ} \mathrm{C}$.

The procedures in the study were approved by Hacettepe University Animal Care and Use Ethics Committee, Ankara, Turkey, approval numbers 2001/08-3. Experiments were conducted in accordance with the Animal Care Guidelines of Hacettepe University.

Experiments were performed in two groups of mice, PTX, and control. In the PTX group, animals were treated with PTX ( $50 \mathrm{mg} / \mathrm{kg}$, i.p., $\mathrm{n}=12$ ) for a week and the control animals received saline $(n=6)$. Animals were anesthetized with ether and phrenic nerves attached to the diaphragm muscles were removed. Ringer's solution had the following composition (in $\mathrm{mm}$ ): $\mathrm{NaCl}$, 135; $\mathrm{KCl}, 5 ; \mathrm{MgCl}_{2}, 1 ; \mathrm{CaCl}_{2}, 2 ; \mathrm{NaHCO}_{3}, 15 ; \mathrm{Na}_{2} \mathrm{HPO}_{4}$, 1 ; and glucose, 11. Muscle preparations were placed vertically in a $50 \mathrm{ml}$ organ bath at $37^{\circ} \mathrm{C}$ and gassed with a mixture of $95 \%$ oxygen $\left(\mathrm{O}_{2}\right)$ and $5 \%$ carbon dioxide $\left(\mathrm{CO}_{2}\right)$. After applying 1-1.5 g resting tension, isometric muscle contractions were recorded via force displacement transducers (FT03, Grass Instruments, Quincy, MA) on a polygraph (Model 79C Grass Instruments, Quincy, MA). Phrenic nerve and diaphragm muscles were stimulated by rectangular electrical pulses via platinum electrodes connected to a stimulator (Model S88 Grass Instruments, Quincy, MA) and a stimulus isolation unit (SIU5 Grass Instruments, Quincy, MA). A pair of platinum electrodes was placed vertically to both sides of the muscle fibers for direct stimulation. The phrenic nerve was placed between two parallel platinum ring electrodes, which were placed in a polyethylene tubing of $3 \mathrm{~mm}$ diameter. Contraction amplitudes of muscles were normalized and expressed as $\mathrm{g} / \mathrm{g}$ of wet tissue. In order to induce hypoxia, $95 \%$ nitrogen $\left(\mathrm{N}_{2}\right)+5 \%$ carbon dioxide $\left(\mathrm{CO}_{2}\right)$ gas mixture was applied. Isometric muscle contractions, time to reach complete neuromuscular blockade during hypoxia, and the rate of recovery of muscle contractility upon re-oxygenation were determined. Electrophysiological experiments were made by using conventional microelectrode techniques. Muscle preparations were placed on perspex plates covered with Sylgard, the thoracic side on top and were perfused with Ringer's solution $1-2 \mathrm{ml} / \mathrm{min}$ at $22 \pm 1^{\circ} \mathrm{C}$. Muscle preparations were illuminated from the bottom of the organ bath and were observed by a stereomicroscope (Bausch and Lomb). Resting membrane potentials (RMPs), miniature end plate potentials (MEPP), and action potentials (APs) were recorded with borosilicate glass microelectrodes having 10-30 $\mathrm{M} \Omega$ of input resistances filled with $3 \mathrm{~mol} / \mathrm{I} \mathrm{KCl}$. Signals were amplified by Axoclamp 2A (Axon instruments, Foster city, CA, USA) amplifier and displayed on a digital storage oscilloscope (Hitachi VC-6524, Hitachi Denshi, Tokyo, Japan). Amplitude and frequency of MEPPs, threshold level, overshoot, amplitude, and half-rise and half-decay times of APs were evaluated and digitized by Powerlab 8/P (ADI Instruments, Pty. Ltd., Castle Hill, Australia) and Scope 3.2 and Chart 4.0 software programs were used to analyze data.

The following drugs were used: Pentoxifylline (Trental, Roche Istanbul, Turkey) and caffeine (Sigma Chemical Co., MO, and USA).
All data were expressed as mean \pm SEM. Comparison of the groups was done by the Mann-Whitney U-test or two-way ANOVA followed by Bonferroni's post hoc test. Statistical significance was defined as $P<0.05$.

\section{Results}

PTX treatment increased contractility of the diaphragm muscles. Amplitudes of direct and indirect isometric muscle contractions in the PTX-treated group were greater than controls. Diaphragm muscles prepared from the control animals had twitch contraction amplitudes of $22.2 \pm 4.5 \mathrm{~g} / \mathrm{g}$ and $10.0 \pm 1.4 \mathrm{~g} / \mathrm{g}$, upon direct and indirect stimulation, respectively. Muscles obtained from PTX-treated animals displayed about $90 \%$ greater contraction amplitudes for both types of stimulation [Figure $1, P<0.01$ ].

Hypoxia inhibited muscle contractions, caused neuromuscular blockade, and induced a reversible muscle contracture in both groups. Effects of hypoxia were more pronounced in the PTX group. Complete neuromuscular blockade occurred $28.5 \pm 5.7 \mathrm{~min}$ after the commencement of hypoxia, in the control group. However, in the PTX group, this effect occurred $22.5 \pm 6$ min later [Figure $2 a, P<0.05]$. The hypoxia-induced contracture amplitude in the PTX group was significantly greater than the controls, $7.3 \pm 0.5$ and $2.1 \pm 1.1 \mathrm{~g} / \mathrm{g}$ in the PTX-treated and controls, respectively [Figure $2 \mathrm{~b}, P<0.05$ ].

Re-oxygenation was instated in all preparations after completion of neuromuscular transmission failure. During $30 \mathrm{~min}$ of re-oxygenation, indirect muscle twitches reappeared, hypoxic contracture returned to the baseline, and muscle contractility partially recovered [Figure 3]. Indirect muscle twitches reappeared $1.4 \pm 0.2 \mathrm{~min}$ after re-oxygenation in the control group and $1.8 \pm 0.2$ min later in the PTX group $[P<0.05$, Figure 4$]$. In this period, amplitudes of direct and indirect contractions of the diaphragm muscles prepared from the PTX-treated mice were significantly greater $[P<0.01$, Figure 3]. However, the rate of recovery of muscle contractility upon re-oxygenation was slower in the PTX group. At the end of the re-oxygenation period, direct muscle contractions in the control group recovered to $82 \%$ of the baseline values and indirect contractions recovered to $65 \%$.

Figure 1: Effects of hypoxia on diaphragm muscles obtained from PTX-treated mice. After 15 min of the oxygenation period, hypoxia was instated by $95 \% \mathrm{~N}_{2}+5 \% \mathrm{CO}_{2}$ until neuromuscular blockade occurred (control, $\mathrm{n}=6$ and PTX, $\mathrm{n}=12,{ }^{*} P<0.01$ ); $P<0.01$, two-way ANOVA, post hoc Bonferroni

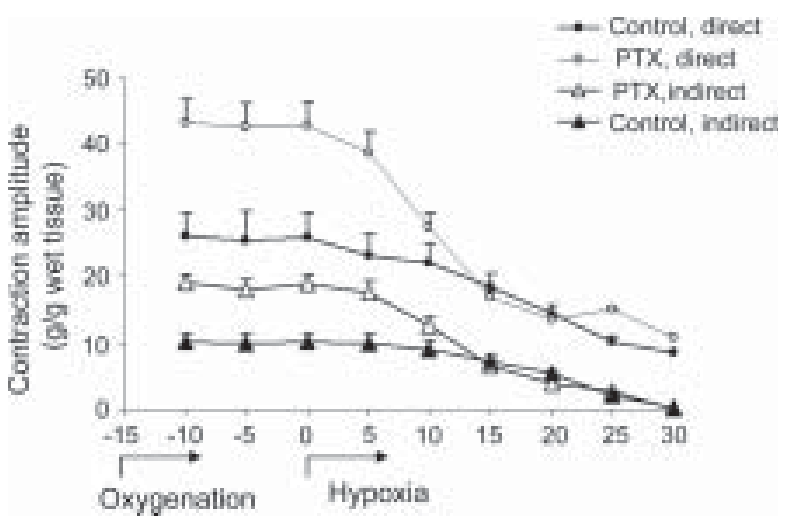


Figure 2: Effects of hypoxia on time to reach complete neuromuscular blockade (a) and contracture development (b) in muscles of PTX-treated mice (control, $\mathrm{n}=6, \mathrm{PTX}, \mathrm{n}=12,{ }^{*} P<0.05$ ); $P<0.05$, the Mann-Whitney U-test

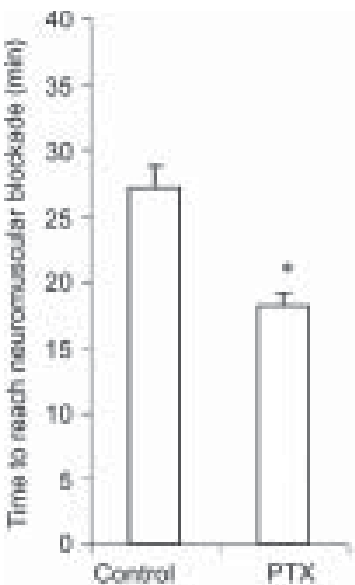

(a) (b)

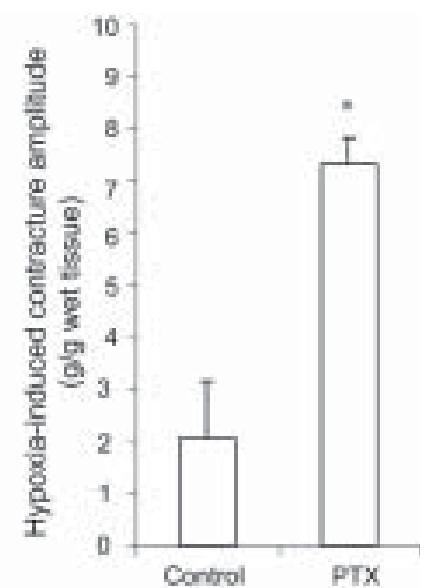

Figure 4: Reappearance of indirect muscle twitches in PTX-treated mice during re-oxygenation (control, $\mathrm{n}=6, \mathrm{PTX}$-treated, $\mathrm{n}=12$, $\left.{ }^{*} P<0.05\right) ; P<0.05$, the Mann-Whitney U-test

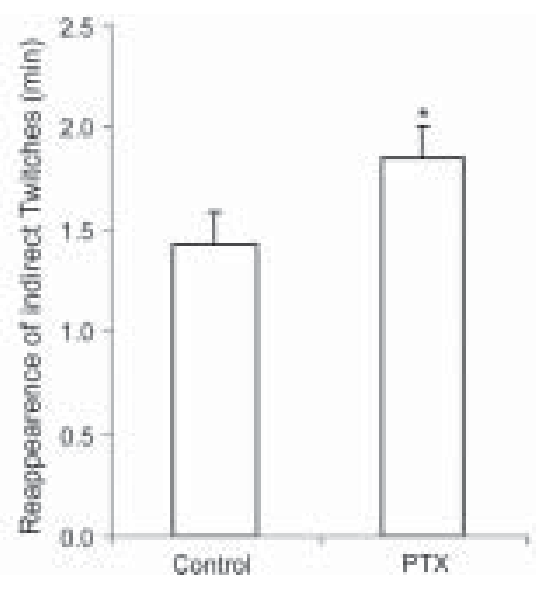

These ratios in the PTX group were $63 \%$ for direct contractions and $61 \%$ for indirect contractions.

PTX treatment did not appreciably alter RMP and spontaneous neurotransmitter release in the mouse diaphragm muscles. Control and PTX-treated muscles had mean MEPP frequencies of $0.96 \pm 0.1$ per $s$ and $0.99 \pm 0.09$ per $s$, and amplitudes of $1.93 \pm 0.11 \mathrm{mV}$ and $1.86 \pm 0.09 \mathrm{mV}$, respectively. RMP of control muscles was $75.30 .3 \mathrm{mV}$ and did not alter in skeletal muscles of PTX-treated mice (74.8 0.4 mV).

PTX treatment significantly altered AP configuration of the diaphragm muscles without changing threshold for excitation. APs recorded from PTX-treated animals were increased in amplitude and had a faster time course. APs of these muscles were about $13 \mathrm{mV}$ greater than that of controls [Figure $5 \mathrm{a}$, $P<0.05]$. Half-rise times of the APs of the PTX-treated muscles were significantly [Figure $5 b, P<0.05$ ] shorter than that of control muscles and found to be $0.30 \pm 0.04 \mathrm{~ms}$
Figure 3: Effects of re-oxygenation in PTX-treated diaphragm muscles. Contractile recovery from hypoxia was investigated for $30 \mathrm{~min}$ in muscle strips in the presence of $95 \% \mathrm{O}_{2}+5 \% \mathrm{CO}_{2}$ (control, $\mathrm{n}=6$ and PTX, $\left.\mathrm{n}=12,{ }^{*} P<0.01\right) ; P<0.01$, two-way ANOVA, post hoc Bonferroni

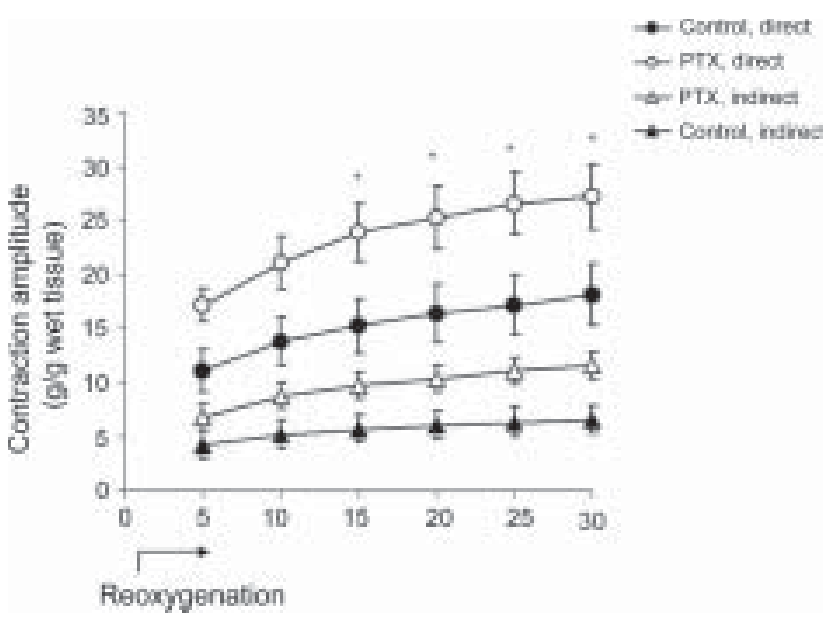

and $0.38 \pm 0.07 \mathrm{~ms}$, respectively. Half-decay times of the PTX-treated group were also shorter than the control group $(0.60 \pm 0.07 \mathrm{~ms}$ and $0.71 \pm 0.05 \mathrm{~ms}$, respectively); however, this difference did not reach statistical significance.

\section{Discussion}

This study demonstrated that chronic PTX administration increased twitch and AP amplitudes of diaphragm muscle, in mice. PTX treatment shortened time course of muscle APs without altering RMP, excitation threshold, and spontaneous neurotransmitter release. Contractile impairment in hypoxia was more pronounced with prolonged recovery upon re-oxygenation.

One of the mechanisms involved in the increase in contractility observed in PTX-treated animals appears to be related to PDE inhibition, which alters mechanisms related to cytosolic $\mathrm{Ca}^{2+}$ handling. ${ }^{[9,10]}$ In our study, PTX-treated diaphragm muscles did not display alteration of $\mathrm{KCl}$ and caffeine contractures, implying that PTX at this dose level did not change depolarization-contraction coupling and $\mathrm{Ca}^{2+}$ release from the sarcoplasmic reticulum (SR).

Regulatory effects of PTX on cytokine expression may contribute to increased skeletal muscle contractility. PTX decreases the expression of TNF- $\alpha$ in various tissues, including the skeletal muscle. [5] PTX also inhibits cellular TNF- $\alpha$ synthesis and attenuates toxic effects of TNF- $\alpha{ }^{[4,11]}$ It is shown that cytokines, predominantly TNF- $\alpha$, have a prominent role in skeletal muscle contractile dysfunction in these cytokine-related pathologies. ${ }^{[12]}$ In a recent study, Alloatti et al. showed that TNF- $\alpha$ reduced contractility and shifted the force-frequency relationship to the right in guinea pig extensor digitorum longus muscle. They also found that the TNF- $\alpha$-induced effect is mediated by NO production, which is induced by a plateletactivating factor. ${ }^{[13]}$ In another study, TNF- $\alpha$-induced reduction of contractile force in cardiac myocytes was found to be dependent on excessive NO production. ${ }^{[6]} \mathrm{NO}$ production impairs contractile response in the skeletal muscle by acting on intracellular $\mathrm{Ca}^{2+}$ 
Figure 5: Effects of PTX treatment on excitation threshold overshoot and action potential amplitudes; (a) (control, $n=4,40$ muscle fibers; PTX-treated $n=7,40$ muscle fibers) half rise and half decay times recorded from diaphragm muscles by indirect stimulation; (b) (control, $n=4$ muscles and 59 fibers; PTX-treated, $\mathrm{n}=12$ muscles and 40 fibers, ${ }^{*} P<0.05$ ); $P<0.05$, the Mann-Whitney $U$-test

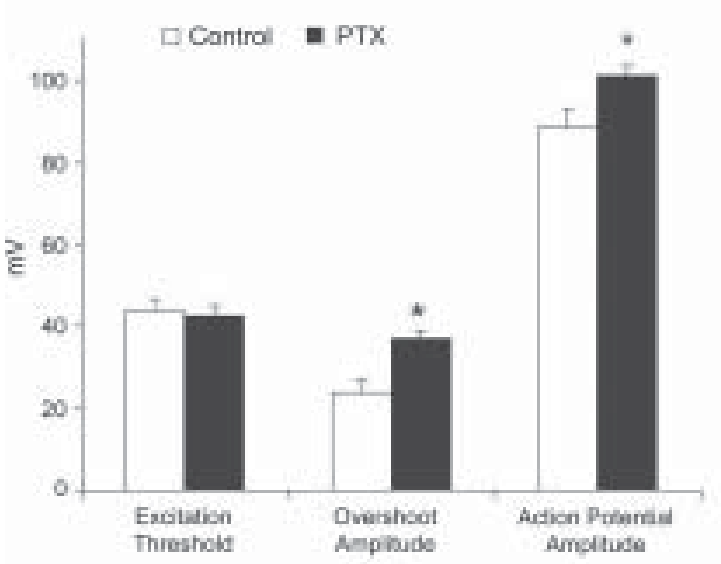

(a)

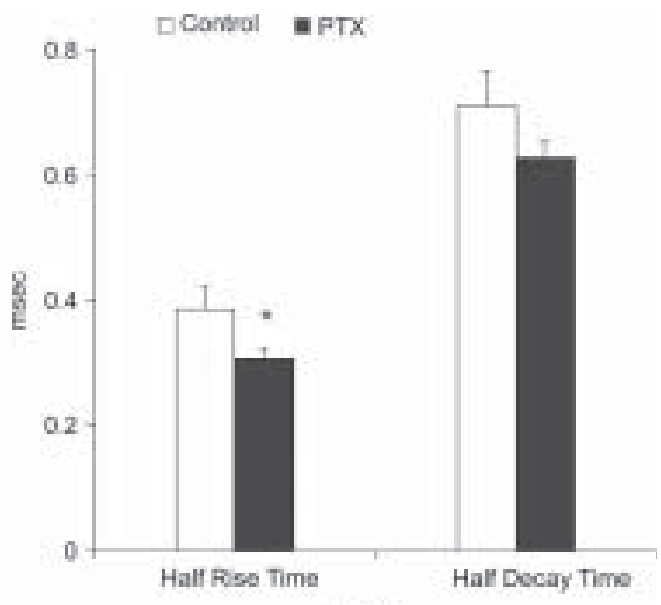

(b) transients, SR Ca ${ }^{2+}$ ATP-ase expression, and/or $\mathrm{Ca}^{2+}$ release channel. ${ }^{[14]}$ We found that muscles obtained from PTX-treated mice had greater twitch amplitudes in normoxic conditions, however, in the hypoxic state; the contractility of PTX-treated muscles was lower than that of controls. This may be related to the diverse effects of PTX on basal and hypoxia-induced local expression of cytokines, specifically, to TNF- $\alpha$ and IL- 6 which have recently been shown to induce various effects on skeletal muscle protein metabolism, proliferation, NO production, and contractility. ${ }^{[15]}$ We also found that in the re-oxygenation period, amplitudes of direct and indirect twitch contractions of PTX-treated muscles were greater, indicating a more efficient recovery process. Greater contractile responses obtained in our study in PTX-treated muscles may be explained in part, by NOS inhibition and/or decreased NO production.

Detrimental effects of hypoxia on contractility were increased in muscles obtained from PTX-treated animals. This is evidenced by the findings that hypoxia-induced twitch amplitude inhibition and contracture were greater in PTX-treated muscles. Also recovery upon re-oxygenation was delayed in these muscles. These effects may be due to increased $\mathrm{Ca}^{2+}$ influx after PDE inhibition by PTX treatment. Excessive amounts of cytosolic $\mathrm{Ca}^{2+}$ is deleterious, and it has been shown that elevated $\mathrm{Ca}^{2+}$ induces disruption of the excitation-contraction (E-C) coupling. ${ }^{[16]}$ Increased intracellular $\mathrm{Ca}^{2+}$ concentration activates proteases and phospholipases, which contribute to the damage in the mitochondria and SR. ${ }^{[17-19]}$ Damage of these organelles eventually causes progression of the deterioration by further increasing intracellular $\mathrm{Ca}^{2+}$ levels. Hypoxia is one of the triggering events initiating this vicious cycle by decreased ATP content to maintain intracellular $\mathrm{Ca}^{2+}$ balance. ${ }^{[20]}$

Our data showed that chronic PTX treatment did not alter RMP of diaphragm muscle and spontaneous neurotransmitter release at the neuromuscular junction. However, PTX treatment significantly increased muscle AP amplitudes and shortened their time course. The reduction in time course was found to be more prominent in the rising phase, implying increased $\mathrm{Na}^{+}$channel activity. PDE inhibitors are reported to alter electrical activity of cardiac and skeletal muscles. Toborinone, a PDE inhibitor by increasing CAMP concentration increased contractility and prolonged AP duration in cardiac myocytes by blocking the delayed rectifier currents. ${ }^{[2]}$ We observed an increase in skeletal muscle contractility concomitant with reduction in AP duration. This difference in AP duration may be due to different types of muscles and drugs used. Recently, Gonzalez-Serratos et al. showed that a different PDE inhibitor, LASSBio-294, did not alter RMP and APs in frog sartorius muscles; however it improved contractility and reduced muscle fatigue. ${ }^{[22]}$

\section{Conclusion}

Chronic PTX treatment increases skeletal muscle contractility without altering spontaneous acetylcholine release, but does not improve hypoxia-induced skeletal muscle dysfunction. These results may implicate important clinical consequences for PTX, a drug that is mainly used for indications related to tissue hypoxia.

\section{References}

1. Brotto MA, Andreatta-van Leyen S, Nosek CM, Brotto LS, Nosek TM. Hypoxia and fatigue-induced modification of function and proteins in intact and skinned murine diaphragm muscle. Pflugers Arch 2000;440:727-34

2. Stellin GP, Waxman K. Current and potential therapeutic effects of pentoxifylline. Compr Ther 1989;15:11-3.

3. Losavio A, Muchnik S. Facilitation of spontaneous acetylcholine release induced by activation of CAMP in rat neuromuscular junctions. Life Sci 2000;66:2543-56.

4. Van Furth AM, Verhard-Seijmonsbergen EM, Van Furth R, Langermans JA. Effect of lisofylline and pentoxifylline on the bacterial-stimulated production of TNF-alpha, IL-1 beta IL-10 by human leucocytes. Immunology 1997;91:193-6.

5. Park E, Schuller-Levis G, Park SY, Jia JH, Levis WR. Pentoxifylline downregulates nitric oxide and tumor necrosis factor-alpha induced by mycobacterial lipoarabinomannan in a macrophage cell line. Int J Lepr Other Mycobact Dis 2001;69:225-33.

6. Finkel MS, Oddis CV, Jacob TD, Watkins SC, Hattler BG, Simmons RL. Negative inotropic effects of cytokines on the heart mediated by nitric oxide. Science 1992;257:387-9.

7. Ralevic V. Hypoxic vasodilatation: Is an adenosine-prostaglandins- NO signalling cascade involved J Physiol 2002;544:2.

8. Kishi M, Tanaka H, Seiyama A, Takaoka M, Matsuoka T, Yoshioka T, et al. 
Pentoxifylline attenuates reperfusion injury in skeletal muscle after partial ischemia. Am J Physiol 1998;274: H1435-42.

9. Ferrier GR, Howlett SE. Differential effects of phosphodiesterase-sensitive and-resistant analogs of CAMP on initiation of contraction in cardiac ventricular myocytes. J Pharmacol Exp Ther 2003;306:166-78.

10. Muniz J, Huerta M, Duenas J, Trujillo X, Elizalde A. Caffeine and theophylline contractures in tonic skeletal muscle fibers of the frog. Jpn J Physiol 1992;42:711-20.

11. Costelli P, Bossola M, Muscaritoli M, Grieco G, Bonelli G, Bellantone R, et al. Anticytokine treatment prevents the increase in the activity of ATP-ubiquitin- and $\mathrm{Ca}\left(2^{+}\right)$-dependent proteolytic systems in the muscle of tumour-bearing rats. Cytokine 2002;19:1-5.

12. Reid MB, Li YP. Cytokines and oxidative signalling in skeletal muscle. Acta Physiol Scand 2001:171:225-32.

13. Alloatti G, Penna C, Mariano F, Camussi G. Role of NO and PAF in the impairment of skeletal muscle contractility induced by TNF-alpha. Am J Physiol Regul Integr Comp Physiol 2000;279: R2156-63.

14. Stamler JS, Meissner G. Physiology of nitric oxide in skeletal muscle. Physiol Rev 2001;81:209-37.

15. Helge JW, Stallknecht B, Pedersen BK, Galbo H, Kiens B, Richter EA. The effect of graded exercise on IL-6 release and glucose uptake in human skeletal muscle.
J Physiol 2003;546:299-305.

16. Verburg E, Dutka TL, Lamb GD. Long-lasting muscle fatigue: Partial disruption of excitation-contraction coupling by elevated cytosolic $\mathrm{Ca}^{2+}$ concentration during contractions. Am J Physiol Cell Physiol 2006;290: C1199-208.

17. Bossenmeyer-Pourie C, Koziel V, Daval JL. CPP32/CASPASE-3-like proteases in hypoxia-induced apoptosis in developing brain neurons. Brain Res Mol Brain Res 1999;71:225-37.

18. Petry C, Huwiler A, Eberhardt W, Kaszkin M, Pfeilschifter J. Hypoxia increases group IIA phospholipase A(2) expression under inflammatory conditions in rat renal mesangial cells. J Am Soc Nephrol 2005;16:2897-905.

19. Edelstein CL. Calcium-mediated proximal tubular injury-what is the role of cysteine proteases Nephrol Dial Transplant 2000;15:141-4.

20. Bannister NJ, Publicover SJ. Interacting effects of $\mathrm{Ca}^{2+}$ and hypoxia in the induction of sarcolemmal damage in mouse diaphragm in vitro. Acta neuropathologica 1995;90:411-4.

21. MacGowan GA. Clinical overview of the novel inotropic agent toborinone. Expert Opin Investig Drugs 2000;9:1109-17.

22. Gonzalez-Serratos H, Chang R, Pereira EF, Castro NG, Aracava Y, Melo PA, et al. A novel thienylhydrazone, (2-thienylidene)3,4-methylenedioxybenzoylhydrazine, increases inotropism and decreases fatigue of skeletal muscle. J Pharmacol Exp Ther 2001;299:558-66.

\section{Dispatch and return notification by E-mail}

The journal now sends email notification to its members on dispatch of a print issue. The notification is sent to those members who have provided their email address to the association/journal office. The email alerts you about an outdated address and return of issue due to incomplete/incorrect address.

If you wish to receive such email notification, please send your email along with the membership number and full mailing address to the editorial office by email. 